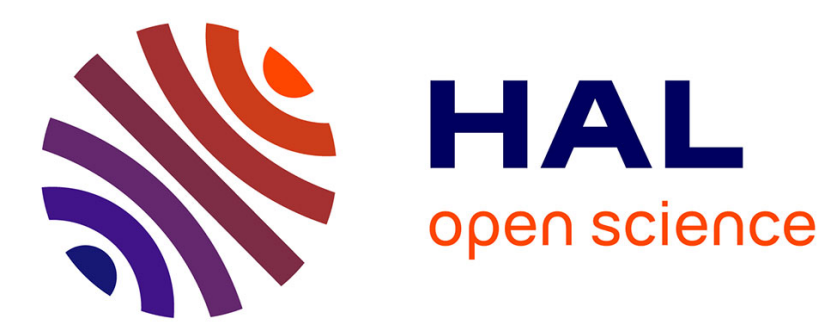

\title{
GAPS FOR GEOMETRIC GENERA
}

C Ciliberto, F Flamini, M Zaidenberg

\section{To cite this version:}

C Ciliberto, F Flamini, M Zaidenberg. GAPS FOR GEOMETRIC GENERA. Archiv der Mathematik, 2016, 106 (6), pp.531-541. 10.1007/s00013-016-0908-0 . hal-01261881

\section{HAL Id: hal-01261881 \\ https://hal.science/hal-01261881}

Submitted on 25 Jan 2016

HAL is a multi-disciplinary open access archive for the deposit and dissemination of scientific research documents, whether they are published or not. The documents may come from teaching and research institutions in France or abroad, or from public or private research centers.
L'archive ouverte pluridisciplinaire HAL, est destinée au dépôt et à la diffusion de documents scientifiques de niveau recherche, publiés ou non, émanant des établissements d'enseignement et de recherche français ou étrangers, des laboratoires publics ou privés. 


\title{
GAPS FOR GEOMETRIC GENERA
}

\author{
C. CILIBERTO, F. FLAMINI, M. ZAIDENBERG
}

\begin{abstract}
We investigate the possible values for geometric genera of subvarieties in a smooth projective variety. Values which are not attained are called gaps. For curves on a very general surface in $\mathbb{P}^{3}$, the initial gap interval was found by $\mathrm{Xu}$ (see [7]), and the next one in our previous paper [4, where also the finiteness of the set of gaps was established and an asymptotic upper bound of this set was found. In the present paper we extend some of these results to smooth projective varieties of arbitrary dimension using a different approach.
\end{abstract}

\section{INTRODUCTION}

We consider the following problem. Let $X$ be a smooth complex projective variety of dimension $n>1$, with an ample divisor $L$. For each positive integer $s<n$, describe the set $\mathcal{P}_{X, s}$ of geometric genera of irreducible subvarieties $V \subset X$ of dimension $s$, and, in particular, the subset $\mathcal{P}_{X, L, s} \subseteq \mathcal{P}_{X, s}$ of geometric genera of irreducible complete intersections of $n-s$ hypersurfaces from $\bigcup_{m \geqslant 1}|m L|$. The complement of each of these sets in $\{0\} \cup \mathbb{N}$ is the corresponding set of $s$-gaps, and its maximal intervals are called $s$-gap intervals. For curves on a very general surface $X$ in $\mathbb{P}^{3}$ of degree $d$ (i.e., $n=2, s=1$ ) with a natural polarization $\mathcal{O}_{X}(1)$ the two sets $\mathcal{P}_{X, \mathcal{O}_{X}(1), 1}$ and $\mathcal{P}_{X, 1}$ coincide; the initial gap interval was found in [7] and the next one in [4. In this case there exists a maximum $G_{d}$ for the set of gaps (4, Thm. 2.4] and Remark 3.2 below). This means that a very general surface of degree $d$ in $\mathbb{P}^{3}$ carries a curve of geometric genus $g$ for any $g>G_{d}$. In the present note we show that the latter remains true for any smooth projective variety, and in particular, for any (not just for a very general) smooth surface of degree $d$ in $\mathbb{P}^{3}$. One of our main results is the following:

Theorem 0.1. Let $X$ be an irreducible, smooth, projective variety of dimension $n>1$, let $L$ be a very ample divisor on $X$ and let $s \in\{1, \ldots, n-1\}$. Then there is an integer $p_{X, L, s}$ (depending on $X, L$ and s) such that for any $p \geqslant p_{X, L, s}$ one can find an irreducible subvariety $Y$ of $X$ of dimension $s$ with at most ordinary points of multiplicity $s+1$ as singularities such that $p_{g}(Y)=p$. Moreover, one can choose $Y$ to be a complete intersection $Y=D_{1} \cap \ldots \cap D_{n-s}$, where $D_{i} \in|L|$ for $i=1, \ldots, n-s-1$ are smooth and transversal and $D_{n-s} \in|m L|$ for some $m \geqslant 1$ is such that $Y$ has ordinary singularities of multiplicity $s+1$.

Let $Y$ be an irreducible variety of dimension $s$. A point $y \in Y$ is ordinary of multiplicity $m(m>1)$, if (i) the Zariski tangent space of $Y$ at $y$ has dimension $s+1$, and

(ii) the (affine) tangent cone to $Y$ at $y$ is a cone with vertex $y$ over a smooth hypersurface of degree $m$ in $\mathbb{P}^{s}$.

An ordinary point of $Y$ is an isolated hypersurface singularity, hence, it is Gorenstein.

The proof of Theorem 0.1 is done in Section 1. In Section 2 we deduce an effective upper bound for gaps in the surface case. In Section 3, we focus on smooth surfaces in $\mathbb{P}^{3}$, proving in particular that in this case there is no absolute gap for geometric genera of curves. That is, for all $d>0$, all non-negative integers are geometric genera for some curves lying on some smooth surfaces of degree $d$ in $\mathbb{P}^{3}$.

Acknowledgments: The first and second authors have been supported by the Italian MIUR Project protocol 2010S47ARA_005 and by GNSAGA of INdAM. The third author was supported by the French-Italian cooperation project GRIFGA and by INdAM. The authors thank all Institutions which helped them in this collaboration, including their own Departments.

2010 Mathematics Subject Classification: 14N25, 14J70, 32J25, 32Q45. Key words: projective hypersurface, geometric genus. 
Notation and conventions. We work over the field of complex numbers and use standard notation and terminology. In particular, for $X$ a reduced, irreducible, projective variety, we denote by $\omega_{X}$ its dualizing sheaf. We will sometimes abuse notation and use the same symbol to denote a divisor $D$ on $X$ and its class in $\operatorname{Pic}(X)$. Thus $K_{X}$ will denote a canonical divisor or the canonical sheaf $\omega_{X}$. When $Y \subset X$ is a closed subscheme, $\mathcal{I}_{Y / X}$ will denote its ideal sheaf.

\section{UPPER BOUND FOR GAPS}

1.1. Preliminaries. In the sequel, $X$ is an irreducible, complex projective variety of dimension $n \geqslant 2$. We assume usually that $X$ is Gorenstein, so that $\omega_{X}$ is a line bundle. This holds, in particular, if $X$ has only ordinary singularities. We set

$$
p(X):=h^{0}\left(X, \omega_{X}\right) \text { and } q(X):=h^{1}\left(X, \omega_{X}\right) .
$$

For smooth varieties, both $p(X)$ and $q(X)$ are birational invariants. Note that, if $X$ is a smooth surface, then $q(X)$ is the irregularity of $X$.

The geometric genus of $X$ is defined as

$$
p_{g}(X):=p\left(X^{\prime}\right),
$$

where $X^{\prime} \rightarrow X$ is any desingularization of $X$.

Lemma 1.1. Let $X$ be an irreducible, smooth projective variety of dimension $n$, and let $Y$ be an irreducible, effective divisor on $X$. Assume that $h^{i}\left(X, \omega_{X} \otimes \mathcal{O}_{X}(Y)\right)=0$ for all $i \geqslant 1$ 1 . Then:

(i) one has

$$
p(Y)=h^{0}\left(X, \omega_{X} \otimes \mathcal{O}_{X}(Y)\right)+q(X)-p_{g}(X),
$$

which is the geometric genus if $Y$ is smooth;

(ii) suppose that $\operatorname{Sing}(Y)=\left\{x_{1}, \ldots, x_{k}\right\}$, where $x_{1}, \ldots, x_{k}$ are ordinary points of $Y$ of multiplicity $n$. Then

$$
p_{g}(Y) \geqslant p(Y)-k,
$$

and the equality holds if and only if $x_{1}, \ldots, x_{k}$ impose $k$ independent conditions to the linear system $\mid \omega_{X} \otimes$ $\mathcal{O}_{X}(Y) \mid$, i.e., if and only if the restriction map

$$
H^{0}\left(X, \omega_{X} \otimes \mathcal{O}_{X}(Y)\right) \longrightarrow \bigoplus_{i=1}^{k} \mathcal{O}_{x_{i}}
$$

is surjective.

Proof. Part (i) follows from the adjunction sequence

$$
0 \longrightarrow \omega_{X} \longrightarrow \omega_{X} \otimes \mathcal{O}_{X}(Y) \longrightarrow \omega_{X} \otimes \mathcal{O}_{X}(Y) \otimes \mathcal{O}_{Y} \cong \omega_{Y} \longrightarrow 0 .
$$

As for part (ii), let $\pi: X^{\prime} \rightarrow X$ be the blow-up of $X$ at $x_{1}, \ldots, x_{k}$ with exceptional divisors $E_{1}, \ldots, E_{k}$. Set $E=\sum_{i=1}^{k} E_{i}$. The union $x$ of $x_{1}, \ldots, x_{k}$ is a 0 -dimensional subscheme of $X$. The proper transform $Y^{\prime}$ of $Y$ in $X^{\prime}$ is smooth and belongs to the linear system $\left|\pi^{*}\left(\mathcal{O}_{X}(Y)\right) \otimes \mathcal{O}_{X^{\prime}}(-n E)\right|$, whereas $\omega_{X^{\prime}}=$ $\pi^{*}\left(\omega_{X}\right) \otimes \mathcal{O}_{X^{\prime}}((n-1) E)$. Hence, by (i), one has

$$
\begin{aligned}
p_{g}(Y)=p_{g}\left(Y^{\prime}\right) & =h^{0}\left(X^{\prime}, \omega_{X^{\prime}} \otimes \mathcal{O}_{X^{\prime}}\left(Y^{\prime}\right)\right)+q\left(X^{\prime}\right)-p_{g}\left(X^{\prime}\right) \\
& =h^{0}\left(X^{\prime}, \pi^{*}\left(\omega_{X} \otimes \mathcal{O}_{X}(Y)\right) \otimes \mathcal{O}_{X^{\prime}}(-E)\right)+q(X)-p_{g}(X) \\
& =h^{0}\left(X, \omega_{X} \otimes \mathcal{O}_{X}(Y) \otimes \mathcal{I}_{x / X}\right)+q(X)-p_{g}(X) .
\end{aligned}
$$

Now the assertion follows.

Lemma 1.2. Let $X \subset \mathbb{P}^{r}$ be a non-degenerate, irreducible projective variety of dimension $n$. Let $x_{1}, \ldots, x_{k} \in$ $X$ be general points. If $k \leqslant r-n=\operatorname{codim}_{\mathbb{P}^{r}}(X)$, then the scheme theoretical intersection of the linear space $\left\langle x_{1}, \ldots, x_{k}\right\rangle$ with $X$ is the reduced 0 -dimensional scheme consisting of $x_{1}, \ldots, x_{k}$.

\footnotetext{
${ }^{1}$ By the Kawamata-Viehweg vanishing theorem this holds provided $Y$ is nef and big (in particular, for $Y$ ample).
} 
Proof. The assertion is trivial for $k=1$, so we assume $k \geqslant 2$. For $n=1$ and $k=2$, this is the classical trisecant lemma, to the effect that a general chord of a non-degenerate curve in $\mathbb{P}^{r}$, where $r \geqslant 3$, is not a trisecant (see, e.g., [1, Example 1.8] for a simple proof). If $n=1$ and $k>2$, one proceeds by applying induction on $k$ to the projection of $X$ to $\mathbb{P}^{r-1}$ from one of the points $x_{1}, \ldots, x_{k}$.

If $n>1$, one proceeds by applying induction on $n$ to the section of $X$ with a general hyperplane containing $\left\langle x_{1}, \ldots, x_{k}\right\rangle$.

\subsection{The theorem.}

Theorem 1.3. Let $X$ be an irreducible, smooth, projective variety of dimension $n>1$, and let $L$ be a very ample line bundle on $X$. Then there is an integer $p_{X, L}$ (depending on $X$ and $L$ ) such that for all $p \geqslant p_{X, L}$ one can find an irreducible hypersurface $Y \in \bigcup_{m \geqslant 1}|m L|$ with at most ordinary points of multiplicity $n$ as singularities and with $p_{g}(Y)=p$.

Proof. Set $d:=L^{n}$. For a positive integer $m$ we denote by $p_{m}$ the geometric genus of smooth elements in $|m L|$ (which is of course a non-gap). We show that for $m$ sufficiently large, any integer $p$ in the interval $\left[p_{m-1}+1, p_{m}-1\right]$ is the geometric genus of a hypersurface in $|m L|$ with $p_{m}-p$ ordinary points of multiplicity $n$ as singularities, which can be taken generically on $X$.

Since $L$ is very ample, by Lemma $1.1-(i)$ and by the asymptotic Riemann-Roch Theorem [6, Vol. I, p. 21], we have

$$
\begin{aligned}
p_{m} & =\chi\left(\omega_{X} \otimes \mathcal{O}_{X}(m L)\right)+q(X)-p_{g}(X) \\
& =h^{0}\left(\omega_{X} \otimes \mathcal{O}_{X}(m L)\right)+q(X)-p_{g}(X) \\
& =\frac{m^{n}}{n !} d+O\left(m^{n-1}\right) .
\end{aligned}
$$

Hence

$$
\delta_{m}:=p_{m}-p_{m-1}-1=\frac{m^{n-1}}{(n-1) !} d+O\left(m^{n-2}\right) .
$$

Theorem 1.3 follows from the:

Claim 1. There is an integer $m_{X, L}$ (depending on $X$ and $L$ ) such that for all $m \geqslant m_{X, L}$, for all positive integers $k \leqslant \delta_{m}$, and for general points $x_{1}, \ldots x_{k}$ in $X$, one can find an irreducible element $Y \in|m L|$ with ordinary points of multiplicity $n$ at $x_{1}, \ldots, x_{k}$ and no other singularity.

Indeed, suppose that Claim 1 holds. Then the map (1) is surjective by the generality of $x_{1}, \ldots, x_{k}$. Thus Lemma 1.1-(ii) implies Theorem 1.3 with

$$
p_{X, L}:=p_{m_{X, L}-1}
$$

In turn, Claim 1 is a consequence of the following

Claim 2. There is an integer $m_{X, L} \geqslant n$ such that for all $m \geqslant m_{X, L}$, one has

$$
\delta_{m} \leqslant \operatorname{dim}(|\nu L|)-n, \quad \text { where } m=n \nu+\mu \quad \text { with } \quad \mu \in\{0, \ldots, n-1\} .
$$

Indeed, assuming that Claim 2 holds, let $x$ be the reduced 0-dimensional scheme formed by the points $x_{1}, \ldots, x_{k}$, and let $\Lambda:=\nu L \otimes \mathcal{I}_{x / X}$. By Lemma 1.2, (44) ensures that $x$ is the base locus scheme of the linear system $|\Lambda|$. Therefore, by Bertini's theorem the general $Y \in\left|\Lambda^{\otimes n} \otimes \mathcal{O}_{X}(\mu)\right| \subset|m L|$ is irreducible having $x_{1}, \ldots, x_{k}$ as ordinary points of multiplicity $n$ and no other singularity. Thus, Claim 2 implies Claim 1.

Finally, we prove Claim 2.

Proof of Claim 2. By the asymptotic Riemann-Roch Theorem (cf. (2)), one has

$$
\operatorname{dim}(|\nu L|)=\frac{\nu^{n}}{n !} d+O\left(\nu^{n-1}\right) .
$$

Hence, by (3), Claim 2 holds if, for $m \gg 0$, one has

$$
n m^{n-1}<\nu^{n} \text {. }
$$


Since $\frac{m}{n}<\nu+1$, (15) is true for $m \gg 0$.

This ends the proof of Theorem 1.3 .

Remark 1.4. As follows from the proof, the upper bound $p_{X, L}$ depends only on the Hilbert polynomial of $\bigoplus_{m \geqslant 1} H^{0}\left(X, \omega_{X} \otimes \mathcal{O}_{X}(m L)\right)$ and of $\bigoplus_{m \geqslant 1} H^{0}\left(X, \mathcal{O}_{X}(m L)\right)$. The former coincides with the Hilbert function by Kodaira's Theorem. Assuming that $h^{i}\left(X, \mathcal{O}_{X}(m L)\right)=0$ for all positive integers $m$ and $i$, it is possible to replace the asymptotic Riemann-Roch theorem with the true Riemann-Roch, which is then purely numerical. This gives in principle an effective bound on the integers $m_{X, L}$ and $p_{X, L}$ in Theorem 1.3 (cf. Section 2 for a particular case).

Proof of Theorem 0.1. With $X, L, n$, and $s$ as in Theorem 0.1, it suffices to apply Theorem 1.3 to $X^{\prime}=D_{1} \cap \ldots \cap D_{n-s-1}$ instead of $X$ and $\left.L\right|_{X^{\prime}}$ instead of $L$, where $D_{1}, \ldots, D_{n-s-1} \in|L|$ are general.

\section{Genera of CURVES ON SMOOTh SURFACES}

In this section we compute an effective upper bound for gaps of geometric genera of curves on surfaces.

Theorem 2.1. Let $S$ be a smooth, irreducible, projective surface, and $L$ a very ample line bundle on $S$. Set

$$
p:=p_{g}(S), \quad q:=q(S), \quad d:=L^{2}, \quad \text { and } \quad e:=K_{S} \cdot L .
$$

For $\varepsilon \in\{0,1\}$, set

$$
\begin{aligned}
& \Delta(\varepsilon):=4(3+2 \varepsilon) d^{2}+12 d e+e^{2}-8 d(p-q), \\
& n_{1}=n_{1}(\varepsilon):= \begin{cases}2 & \text { if } \Delta(\varepsilon)<0, \\
\left\lceil 4+\varepsilon+\frac{e}{d}+\sqrt{\frac{\Delta(\varepsilon)}{d^{2}}}\right] & \text { if } \Delta(\varepsilon) \geqslant 0,\end{cases} \\
& n_{2}=n_{2}(\varepsilon):=\left\lceil\frac{6(p-q)+d(1+\varepsilon)+e(2 \varepsilon-1)-12}{e+2 d(1+\varepsilon)}\right\rceil, \\
& n_{3}:=\min \left\{n \in \mathbb{N} \mid\left\lfloor\frac{n}{2}\right\rfloor^{2} d>n d-\frac{d-e}{2}-1\right\} \text {, } \\
& n_{4}:=\min \left\{n \in \mathbb{N} \mid h^{1}\left(S, \mathcal{O}_{S}(n L)\right)=h^{2}\left(S, \mathcal{O}_{S}(n L)\right)=0\right\} \text {, }
\end{aligned}
$$

and

$$
n_{0}=n_{0}(\varepsilon):=\max \left\{n_{1}(\varepsilon), n_{2}(\varepsilon), n_{3}, n_{4}\right\}
$$

Set finally

$$
\varphi\left(d, e, n_{0}\right)=\frac{1}{2}\left[\left(n_{0}-1\right)\left(\left(n_{0}-1\right) d+e\right)\right]+1 .
$$

Then for any $g \geqslant \varphi\left(d, e, n_{0}\right)$ the surface $S$ carries a reduced, irreducible curve $C$ of geometric genus $g$ with only nodes as singularities.

The proof of Theorem 2.1 is basically the same as the one of Theorem 1.3 in the case of surfaces, with a slight improvement, based upon the following:

Theorem 2.2. ([1, Thm. 1.4], [5, Thm. 1.3]) Let $X \subset \mathbb{P}^{r}$ be an irreducible, projective, non-degenerate variety of dimension $m$. Assume $X$ is not $k$-weakly defective for a given $k \geqslant 0$ such that

$$
r \geqslant(m+1)(k+1) \text {. }
$$

Then, given general points $p_{0}, \ldots, p_{k}$ on $X$, the general hyperplane $H$ containing $T_{X, p_{0}, \ldots, p_{k}}{ }^{2}$ is tangent to $X$ only at $p_{0}, \ldots, p_{k}$. Such a hyperplane $H$ cuts out on $X$ a divisor with ordinary double points at $p_{0}, \ldots, p_{k}$ and no further singularities.

\footnotetext{
${ }^{2} T_{X, p_{0}, \ldots, p_{k}}$ stands for the linear span of the union of the embedded tangent spaces $T_{X, p_{i}}, i=0, \ldots, k$.
} 
Recall (see [3, p. 152]) that a variety $X$ as in Theorem [2.2 is said to be $k$-weakly defective if, given $p_{0}, \ldots, p_{k} \in X$ general points and a general hyperplane $H$ containing $T_{X, p_{0}, \ldots, p_{k}}$ (i.e., tangent to $X$ at $\left.p_{0}, \ldots, p_{k}\right)$, then $H$ cuts out on $X$ a divisor $H_{X}$ such that there is a positive dimensional subvariety $\Sigma \subseteq$ $\operatorname{Sing}\left(H_{X}\right)$ containing $p_{0}, \ldots, p_{k}(\Sigma$ is then called the contact variety of $H)$.

2.1. Proof of Theorem 2.1. The arithmetic genus of curves in $|n L|$ is

$$
p(d, e, n):=\frac{1}{2} n(n d+e)+1 \text {. }
$$

For $n \geqslant n_{0}$ set

$$
l(d, e, n):=\operatorname{dim}(|n L|)=\frac{1}{2} n(n d-e)+p-q,
$$

where the latter equality follows by the Riemann-Roch Theorem and (10), since we assume $n \geqslant n_{0} \geqslant n_{4}$. Consider the embedding

$$
\varphi_{|n L|}: S \hookrightarrow \mathbb{P}^{l(d, e, n)} .
$$

Since $\varphi_{|n L|}$ is an isomorphism of $S$ to its image $S_{n}$, we may identify $S$ with $S_{n}$.

Set

$$
\delta(d, e, n):=p(d, e, n)-p(d, e, n-1)-1=n d-\frac{1}{2}(d-e)-1 .
$$

As in the proof of Theorem 1.3, we show that for any $n \geqslant n_{0}$ and any positive integer $k \leqslant \delta(d, e, n)-1$, one can find an irreducible curve $C \in|n L|$ with exactly $k+1$ nodes at general points of $S$ as its only singularities. Then, for any $n \geqslant n_{0}$, all the integers in the interval $J_{n}=[p(d, e, n-1), p(d, e, n)]$ are non-gaps. Since the intervals $J_{n}$ and $J_{n+1}$ overlap, this proves Theorem 2.1, because

$$
\varphi\left(d, e, n_{0}\right):=\min \left(J_{n_{0}}\right)=p\left(d, e, n_{0}-1\right)
$$

is exactly (12).

The proof follows by Proposition 2.3 and Lemma 2.4 below (which are of independent interest).

Proposition 2.3. Let $S$ be a smooth, irreducible, projective surface, and $L$ a very ample line bundle on $S$. Assume that $n \geqslant \max \left\{n_{3}, 2\right\}$ and that (with the above notation) the following inequalities hold

$$
l(d, e, n) \geqslant 3(\delta(d, e, n)-1)
$$

and

$$
l(d, e,\lfloor n / 2\rfloor) \geqslant \delta(d, e, n)+1 .
$$

Then for any $k \in\{0, \ldots, \delta(d, e, n)-1\}$,

(a) the smooth surface $S_{n} \subset \mathbb{P}^{l(d, e, n)}$ is not $k$-weakly defective, and

(b) there exists a reduced, irreducible curve $C \in|n L|$ in $S$ with nodes at $k+1$ general points of $S$ and no other singularity.

Proof. Let $x_{0}, \ldots, x_{k}$ be general points of $S$. Inequality (19) guarantees that, for any $k \in\{0, \ldots, \delta(d, e, n)-$ $1\}$, one has

$$
\operatorname{dim}\left|\mathcal{O}_{S}(\lfloor n / 2\rfloor L) \otimes \mathcal{I}_{\left\{x_{0}, \ldots, x_{k}\right\} / S}\right|=l(d, e,\lfloor n / 2\rfloor)-k-1 \geqslant l(d, e,\lfloor n / 2\rfloor)-\delta(d, e, n) \geqslant 1 .
$$

The general curve in $\left|\mathcal{O}_{S}(\lfloor n / 2\rfloor L) \otimes \mathcal{I}_{\left\{x_{0}, \ldots, x_{k}\right\} / S \mid}\right|$ is reduced and irreducible. Letting $C_{1}$ and $C_{2}$ be two different such general curves, and $C_{0}$ a general member of $L$, we obtain a divisor

$$
C=\varepsilon C_{0}+C_{1}+C_{2} \in \mid \mathcal{O}_{S}(n L) \otimes \mathcal{I}_{T_{S, x_{0}, \ldots, x_{k}} / \mathbb{P}^{l(d, e, n)} \mid,}
$$

where $\varepsilon \in\{0,1\}, \varepsilon \equiv n \bmod 2$. Since $C$ is reduced, with nodes at $x_{0}, \ldots, x_{k}$, this shows that (a) holds.

Now (b) follows. Indeed, since $k+1 \leqslant \delta(d, e, n)$, (18) yields (13) with $m=2$ and $r=l(d, e, n)$. Hence Theorem 2.2 applies, and so, the general curve in $\left|\mathcal{O}_{S}(n L) \otimes \mathcal{I}_{T_{S, x_{0}, \ldots, x_{k}} / \mathbb{P} l(d, e, n)}\right|$ has nodes at $x_{0}, \ldots, x_{k}$ and is elsewhere smooth. This curve is irreducible by Bertini's theorem. Indeed, if $n$ is odd, then $\mid \mathcal{O}_{S}(n L) \otimes$ 
$\mathcal{I}_{T_{S, x_{0}, \ldots, x_{k}} / \mathbb{P} l(d, e, n)} \mid$ has no fixed component and is not composed with a pencil. Assume that $n$ is even. By (91),

$$
C_{1} \cdot C_{2}=\frac{n^{2}}{4} d>\delta(d, e, n) \geqslant k+1
$$

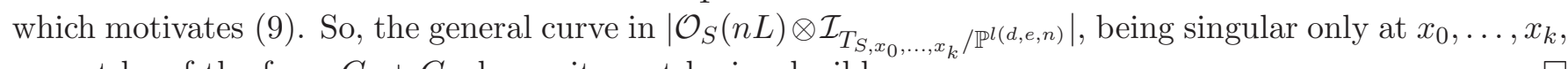
cannot be of the form $C_{1}+C_{2}$, hence it must be irreducible.

Lemma 2.4. Let $\varepsilon \in\{0,1\}$ be such that $\varepsilon \equiv n(\bmod 2)$, and let $\Delta(\varepsilon)$ be as in (6) $)$. Then

(a) (19) holds for any $n \geqslant n_{1}$, with $n_{1}$ as in (7);

(b) if (19) holds, then also (18) holds, provided that $n \geqslant n_{2}$, with $n_{2}$ as in (8).

Proof. (a) Write $n=2 t+\varepsilon$, with $t \geqslant 1$ since $n \geqslant n_{1} \geqslant 2$. From (15) and (16), (19) reads

$$
t^{2} d-t(4 d+e)+2(p-q)-e+(1-2 \varepsilon) d \geqslant 0 .
$$

and the discriminat of the left hand side is $\Delta(\varepsilon)$ as in (6).

When $\Delta(\varepsilon) \geqslant 0$, (20) holds for $t \geqslant \frac{4 d+e+\sqrt{\Delta(\varepsilon)}}{2 d}$, and so (19) holds for

$$
n \geqslant 4+\varepsilon+\frac{e}{d}+\sqrt{\frac{\Delta(\varepsilon)}{d^{2}}} .
$$

If $\Delta(\varepsilon)<0$, then (19) holds for any $n \geqslant 2$. This motivates the definition of $n_{1}$ in (7) and proves (a).

(b) As above, (18) reads

$$
n^{2} d-n(6 d+e)+2(p-q)+3(d-e)+12 \geqslant 0 .
$$

Moreover, (20) reads

$$
n^{2} d-8 n d-2 n e+8(p-q)+4(d-e)+\varepsilon(\varepsilon d-2 n d+2 e) \geqslant 0 .
$$

The difference between the left hand side in (21) and that of (22) is

$$
2 n d+n e-6(p-q)-(d-e)-\varepsilon(\varepsilon d-2 n d+2 e)+12,
$$

which is non-negative as soon as

$$
n \geqslant \frac{6(p-q)+d(1+\varepsilon)+e(2 \varepsilon-1)-12}{e+2 d(1+\varepsilon)} .
$$

Assuming (a), this motivates the definition of $n_{2}$ in (8) and proves (b).

Proof of Theorem 2.1. The integer $n_{0}$ in (111) satisfies both (a) and (b) in Lemma 2.4. Hence (18) and (19) hold, and we can conclude by Proposition 2.3 .

\section{Genera of CURVes on SMooth Surfaces in $\mathbb{P}^{3}$}

Here we focus on the case $S$ is a smooth surface of degree $d \geqslant 4$ in $\mathbb{P}^{3}$. In [4] we considered the case of a very general $S \in\left|\mathcal{O}_{\mathbb{P}^{3}}(d)\right|$; here we drop this assumption, and simply assume $S$ smooth and $d \geqslant 4$ (the case $d<4$ being trivial for our considerations, because then $S$ carries curves of any genus). As a direct consequence of Theorem 2.1, we have:

Corollary 3.1. For any integer $d \geqslant 4$ there exists an integer $c_{d}$ such that, for any smooth surface $S$ in $\mathbb{P}^{3}$ of degree $d$ and any integer $g \geqslant c_{d}, S$ carries a reduced, irreducible nodal curve of geometric genus $g$, whose nodes can be prescribed generically on $S$.

One can give an effective upper bound for $c_{d}$. We keep here the notation of Section 2, Letting $L=\mathcal{O}_{S}(1)$ we obtain

By Theorem 2.1 one has

$$
e=d(d-4), \quad q=q(S)=0, \quad \text { and } \quad p=p_{g}(S)=\frac{1}{6}(d-1)(d-2)(d-3) .
$$

$$
c_{d} \leqslant \varphi\left(d, d(d-4), n_{0}\right)
$$


cf. (12). Thus, we are left to compute $n_{0}$ as in (11). Since, by Serre duality, $n_{4}=d-3$, this amounts to compute $n_{1}, n_{2}$, and $n_{3}$ as in (77), (8)), and (9)).

From (6) we get

$$
\Delta(\varepsilon)=d\left(-\frac{1}{3} d^{3}+12 d^{2}-\frac{1}{3}(104-24 \varepsilon) d+8\right) .
$$

The polynomial $\Delta(\varepsilon) / d$ has three positive roots

$$
d_{1}, d_{2}, d_{3} \sim \begin{cases}0,25,2,89,32,86 & \text { if } \quad \varepsilon=0, \\ 0,36,2, \quad 33,64 & \text { if } \quad \varepsilon=1 .\end{cases}
$$

Thus, $\Delta(0) \geqslant 0$ for $4 \leqslant d \leqslant 32$ and $\Delta(0) \leqslant 0$ for $d \geqslant 33$, while $\Delta(1) \geqslant 0$ for $4 \leqslant d \leqslant 33$ and $\Delta(1) \leqslant 0$ for $d \geqslant 34$.

Now (7), (8), and (9) give, respectively,

$$
\begin{aligned}
& n_{1}(0)=\left\{\begin{array}{ll}
2 & \text { if } d \geqslant 33, \\
\left\lceil d+\sqrt{\frac{\Delta(0)}{d^{2}}}\right] & \text { if } 4 \leqslant d \leqslant 32,
\end{array} \quad n_{1}(1)= \begin{cases}2 & \text { if } d \geqslant 34, \\
\left\lceil d+1+\sqrt{\frac{\Delta(1)}{d^{2}}}\right\rceil & \text { if } 4 \leqslant d \leqslant 33,\end{cases} \right. \\
& n_{2}(0)=\left\lceil d-5+\frac{6(d-3)}{d(d-2)}\right\rceil, \quad n_{2}(1)=\left\lceil d-5+\frac{9(d-2)}{d^{2}}\right\rceil,
\end{aligned}
$$

and

$$
n_{3}(0)=3+\lfloor\sqrt{2 d-6-(4 / d)}\rfloor, \quad n_{3}(1)=4+\lfloor\sqrt{2 d-2-(4 / d)}\rfloor .
$$

In particular, for $d \gg 0$, one has

$$
n_{1}=2, \quad n_{2} \sim d-5, \quad n_{3} \sim \sqrt{d}, \quad \text { hence } \quad n_{0}=n_{4}=d-4 .
$$

So, by (12) and (23),

$$
c_{d} \leqslant \varphi(d, d(d-4), d-4)=\frac{d(d-5)(2 d-9)}{2} \sim d^{3} .
$$

Remark 3.2. Let $\operatorname{Gaps}(d)$ be the set of gaps for geometric genera of irreducible curves on $S \in\left|\mathcal{O}_{\mathbb{P}^{3}}(d)\right|$ very general. By [4, Theorem 2.4], one has

$$
\operatorname{Gaps}(4)=\varnothing, \operatorname{Gaps}(5)=\{0,1,2\}, \quad \text { and } \operatorname{Gaps}(d) \subset\left[0, \frac{d(d-1)(5 d-19)}{6}-1\right] \text { for } d \geqslant 6 .
$$

This is compatible with the results of the present section. A more refined analysis based on [4, Remark 2.5], shows that the maximum $G_{d}$ of $\operatorname{Gaps}(d)$ goes like $G_{d}=O\left(d^{\frac{8}{3}}\right)$. It is an open problem to see if this is sharp.

3.1. Absence of absolute gaps for curves on smooth surfaces in $\mathbb{P}^{3}$. We say that an integer $g$ is a $d$-absolute gap if there is no irreducible curve with geometric genus $g$ on any smooth surface of degree $d$. We show here that there is no absolute gap at all.

Theorem 3.3. For any positive integer $d$ and for any non-negative integer $g$, there is a smooth surface $S \subset \mathbb{P}^{3}$ of degree $d$ and an irreducible, nodal curve $C$ on $S$ with geometric genus $g$.

Proof. We may assume $d \geqslant 5$, otherwise the result is well known (cf. e.g. [4, Prop. 1.2 and Cor. 2.2]).

We set

$$
\ell_{d, n}:=l(d, d(d-4), n)=\left\{\begin{array}{cc}
\frac{n\left(n^{2}+6 n+11\right)}{6} & \text { if } n<d \\
\frac{d\left(3 n^{2}-3 n(d-4)+\left(d^{2}-6 d+11\right)\right)}{6}-1 & \text { if } n \geqslant d
\end{array}\right.
$$

and

$$
p_{d, n}:=p(d, d(d-4), n)=\frac{d n(d+n-4)}{2}+1 .
$$


By [4, Thm. 2.4 and Rem. 2.5], for $S \subset \mathbb{P}^{3}$ very general one has

$$
\operatorname{Gaps}(d) \subset\left[0, p_{d, n-1}-\ell_{d, n-1}-1\right]=\left[0, \frac{d(d-1)(5 d-19)}{6}-1\right] \text { if } d>n \geqslant \sqrt[3]{12 d^{2}} .
$$

Plugging $n=d-1$ in this formula, we obtain the desired result for all

$$
g \geqslant p_{d, d-2}-\ell_{d, d-2} .
$$

Take now $n \leqslant d-2$. By [2, Theorem 3.1], for a general surface $\Sigma \subset \mathbb{P}^{3}$ of degree $n$ with $4 \leqslant n \leqslant d-2$ and for any $g \in\left[p_{n, d}-\ell_{n, d}, p_{n, d}\right]$ there is a reduced, irreducible component $\mathcal{V}$ of the Severi variety of complete intersections of $\Sigma$ with surfaces of degree $d$ having $\delta=p_{n, d}-g$ nodes as the only singularities.

Notice that the union of integers in the non-gap intervals

$$
J_{d-1}(n)=\left[p_{n, d-1}-\ell_{n, d-1}, p_{n, d-1}\right] \text { and } J_{d}(n)=\left[p_{n, d}-\ell_{n, d}, p_{n, d}\right]
$$

is an integer interval for $n \leqslant d-2$. To see this, it suffices to observe that

$$
p_{n, d}-\ell_{n, d} \leqslant p_{n, d-1}+1<p_{n, d} .
$$

The inequality on the right is trivial. To show the other inequality

$$
\ell_{n, d} \geqslant p_{n, d}-p_{n, d-1}-1,
$$

using (24), (25), and the fact that $d \geqslant n+2>n$, we can rewrite it as

$$
3 d(d-n+2)+\left(n^{2}-9 n+26\right) \geqslant 0,
$$

which holds if $d \geqslant n+2$.

Any curve $C \in \mathcal{V}$ is cut out on $\Sigma$ by a surface $S$ of degree $d$. We claim that $S$ can be taken to be smooth. Since the linear system $\left|\mathcal{I}_{C / \mathbb{P}^{3}}(d)\right|$ is base point free outside $C$, by Bertini's theorem $S$ can be choosen to be smooth off $C$. Suppose $S$ is singular at a point $p \in C$. Since $C$ has $\delta$ nodes and no other singularity, and it is the complete intersection of $S$ and $\Sigma$, then $p$ is a node of $C$. But the general surface in $\left|\mathcal{I}_{C / \mathbb{P}^{3}}(d)\right|$ is non-singular at the nodes of $C$, because $\left|\mathcal{I}_{C / \mathbb{P}^{3}}(d)\right|$ contains all surfaces of the form $\Sigma+\Phi$, where $\Phi$ is a general surface of degree $d-n$ (so it does not contain the nodes of $C$ ), and $\Sigma$ is smooth (thus $\Sigma+\Phi$ is smooth at the nodes of $C$ ).

In this way we find nodal curves of any geometric genus $g \geqslant p_{4, d}-\ell_{4, d}=0$ on smooth surfaces of degree $d$, proving the assertion.

Let us conclude by the following conjecture.

Conjecture. For any smooth, rational variety $X$ of dimension $n+1$, any very ample line bundle $L$ on $X$, any $s \in\{1, \ldots, n-1\}$, and any integer $g \geqslant 0$ there is a smooth hypersurface $D \in\left|\mathcal{O}_{X}(L)\right|$ carrying an $s$-dimensional subvariety $S \subset D$ of geometric genus $g$.

In particular, for any $n \geqslant 3, d \geqslant 1, s \in\{1, \ldots, n-1\}$, and $g \geqslant 0$ there is a smooth hypersurface $D \in\left|O_{\mathbb{P}^{n+1}}(d)\right|$ and a subvariety $S \subset D$ as before.

One can ask whether the same holds, more generally, for any smooth Fano variety $X$.

\section{REFERENCES}

[1] L. Chiantini, C. Ciliberto. A few remarks on the lifting problem, Proceedings Conf. of Algebraic Geometry, Paris, 1992, Asterisque 218 (1993), 95-109.

[2] L. Chiantini, C. Ciliberto, On the Severi varieties of surfaces in $\mathbb{P}^{3}$, J. Alg. Geom., 8 (1999), 67-83.

[3] L. Chiantini, C. Ciliberto. Weakly defective varieties, Trans. Amer. Math. Soc. 354 (2001), 151-178.

[4] C. Ciliberto, F. Flamini, M. Zaidenberg. Genera of curves on a very general surface in $\mathbb{P}^{3}$. Intern. Mathem. Research Notes (2015), DOI 10.1093/imrn/rnv055, 20p.

[5] C. Ciliberto, F. Russo. Varieties with minimal secant degree and linear systems of maximal dimension on surfaces. Advances in Mathematics 200 (2006), 1-50.

No. 52. Springer-Verlag, New York-Heidelberg, 1977.

[6] R. Lazarsfeld, Positivity in Algebraic Geometry, voll. I-II, Springer Verlag, 2004.

[7] G. Xu. Subvarieties of general hypersurfaces in projective space. J. Differential Geom. 39(1) (1994), $139-172$. 
Dipartimento di Matematica, Università degli Studi di Roma "Tor Vergata", Via della Ricerca Scientifica, 00133 Roma, ItAly

E-mail address: cilibert@mat.uniroma2.it, flamini@mat.uniroma2.it

Université Grenoble I, Institut Fourier, UMR 5582 CNRS-UJF, BP 74, 38402 Saint Martin D'Hères Cédex, FRANCE

E-mail address: mikhail.zaidenberg@ujf-grenoble.fr 